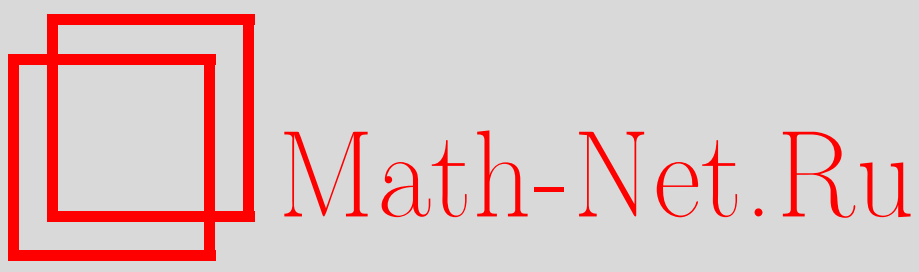

В. М. Мануйлов, А. С. Мищенко, Асимптотические и фредгольмовы представления дискретных групп, Матем. сб., 1998, том 189, номер 10, 53-74

DOI: https://doi.org/10.4213/sm350

Использование Общероссийского математического портала Math-Net.Ru подразумевает, что вы прочитали и согласны с пользовательским соглашением

http://www . mathnet.ru/rus/agreement

Параметры загрузки:

IP: 54.164 .48 .24

26 апреля 2023 г., $18: 28: 23$ 
УДК 517.98

\author{
В.М. Мануйлов, А.С. Мищенко
}

\title{
Асимптотические и фредгольмовы представления дискретных групп
}

\begin{abstract}
Нами построена $C^{*}$-алгебра, обслуживающая теорию асимптотических представлений, и ее вложение в алгебру Калкина, индуцирующее изоморфизм $K_{1}$-групп. Как следствие, мы показьваем, что векторные расслоения над классифицирующим пространством $B \pi$, которые могут быть получены с помощью асимптотических представлений дискретной группы $\pi$, могут быть получены и с помощью представлений группы $\pi \times \mathbb{Z}$ в алгебру Калкина. Мы приводим также обобщение понятия фредгольмова представления и показываем, что асимптотические представления можно рассматривать как асимптотические фредгольмовы представления.

Библиографояи: 8 названий.
\end{abstract}

\section{Введение}

Различные классы представлений дискретных групп оказываются полезньми для сопоставления гомотопических инвариантов неодносвязных многообразий с рациональными характеристическими классами Понтрягина многообразий. Именно, если $\rho: \pi_{1}(X) \rightarrow A$ есть унитарное представление фундаментальной группы $\pi_{1}(X)$ многообразия $X$ в некоторую $C^{*}$-алгебру $A$, то справедлива следуюшая обобщенная формула Хирцебруха:

$$
\operatorname{sign}_{\rho}(X)=2^{2 k}\left\langle L(X) \cdot f_{X}^{*} \operatorname{ch}_{\rho}(\xi),[X]\right\rangle \in K_{0}(A) \otimes \mathbb{Q}
$$

где $\operatorname{sign}_{\rho}(X) \in K_{0}(A)$ есть обобщенная сигнатура многообразия $X$, являющаяся гомотопическим инвариантом, $L(X)$ - класс Хирцебруха многообразия $X$, $[X] \in H_{4 k}(X)$ - его фундаментальньй класс, $\operatorname{dim} X=4 k, f_{X}: X \rightarrow B \pi$ - харак-

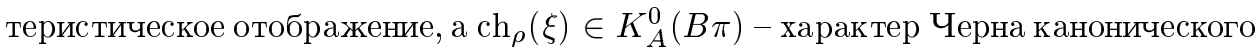
$A$-расслоения над $B \pi$, порожденного представлением $\rho$.

В случае, когда $\rho$ является фредгольмовым представлением или, более общим образом, представлением в алгебру Калкина $\mathscr{Q}$, эта формула дает соотношение числовых инвариантов, поскольку $K_{0}(\mathscr{Q})=0$, а $K_{1}(\mathscr{Q})=\mathbb{Z}$. Другим примером служат так называемые асимптотические представления, изучаемые в настоящей работе, которые тоже дают соотношение числовых инвариантов.

Работа вьполнена при частичной поддержке РФФИ (грант № 96-01-00276) и РФФИ/ННО (грант № 96-01-00028). 
Цель настоящей работы - дать корректное определение асимптотических представлений как представлений в некоторую $C^{*}$-алгебру и показать, что в некотором смысле асимптотические представления являются частным случаем представлений в алгебру Калкина. Как следствие, мы показываем, что класс векторных расслоений над классифицирующим пространством $B \pi$, задаваемых асимптотическими представлениями дискретной группы $\pi$, содержится в классе векторных расслоений над $B \pi$, задаваемых представлениями группы $\pi \times \mathbb{Z}$ в алгебру Калкина. В $\S 2$ мы приводим также обобщение понятия фредгольмова представления и показываем, что асимптотические представления можно рассматривать как асимптотические фредгольмовы представления. В последнем параграфе рассмотрена также связь асимптотических представлений с расширениями $C^{*}$-алгебр.

Мы выражаем признательность Г. Г. Каспарову за полезные советы.

Результаты $\S \S 1$ и 2 получены авторами совместно. Результаты $\S 3$ получены В.М. Мануйловым.

\section{§1. Асимптотические представления как представления в алгебру Калкина}

1.1. Определение асимптотического представления. Пусть $\pi$ - дискретная конечно представленная группа, $F \subset \pi$-конечное подмножество. Обозначим через $U(n)$ унитарную группу размерности $n$ и зафиксируем число $\varepsilon>0$.

ОПРЕДЕЛЕНИЕ 1.1. Отображение $\sigma: \pi \rightarrow U(n)$ называется $\varepsilon$-nочти представлением по отношению к конечному подмножеству $F$, если имеют место следуюшие соотношения:

$$
\begin{gathered}
\sigma\left(g^{-1}\right)=\sigma(g)^{-1} \quad \forall g \in \pi, \\
\|\sigma\|_{F}=\sup \{\|\sigma(g h)-\sigma(g) \sigma(h)\|: g, h, g h \in F\} \leqslant \varepsilon .
\end{gathered}
$$

Пусть $\left\{n_{k}\right\}$ - строго возрастающая последовательность натуральных чисел, $\sigma=\left\{\sigma_{k}: \pi \rightarrow U\left(n_{k}\right)\right\}-$ последовательность $\varepsilon_{k}$-почти представлений по отношению к конечному подмножеству $F \subset \pi$. Мы предполагаем, что группы $U\left(n_{k}\right)$ вложены в группы $U\left(n_{k+1}\right)$ стандартным способом

$$
U\left(n_{k}\right) \ni u \mapsto u \oplus \mathbf{E}_{n_{k+1}-n_{k}} \in U\left(n_{k}\right) \oplus U\left(n_{k+1}-n_{k}\right) \subset U\left(n_{k+1}\right),
$$

что позволяет сравнивать почти представления для различных $k$. Рассмотрим отображения

$$
\sigma_{k} \oplus \mathbf{1}_{\left(n_{k+1}-n_{k}\right)}: \pi \rightarrow U\left(n_{k}\right) \oplus U\left(n_{k+1}-n_{k}\right) \rightarrow U\left(n_{k+1}\right)
$$

которые мы также обозначаем через $\sigma_{k}$. 
ОПРЕДЕЛЕНИЕ 1.2 . Пусть $\sigma=\left\{\sigma_{k}\right\}$ - последовательность $\varepsilon_{k}$-почти представлений. Последовательность $\sigma$ называется асимптотическим представлением группы $\pi$ (по отношению к конечному подмножеству $F$ и к последовательности $\left.\left\{n_{k}\right\}\right)$, если выполнены следуюшие соотношения:

$$
\begin{gathered}
\lim _{k \rightarrow \infty} \varepsilon_{k}=0, \\
\lim _{k \rightarrow \infty} \sup \left\{\left\|\sigma_{k}(g)-\sigma_{k+1}(g)\right\|: g \in F \subset \pi\right\}=0 .
\end{gathered}
$$

Пусть $\left\{m_{k}\right\}$ - другая возрастающая последовательность такая, что $m_{k} \geqslant n_{k}$. Тогда заменяя группы $U\left(n_{k}\right)$ на $U\left(m_{k}\right)$ и отображения $\sigma_{k}$ на

$$
\bar{\sigma}_{k}=\sigma_{k} \oplus \mathbf{E}_{\left(m_{k}-n_{k}\right)}: \pi \rightarrow U\left(n_{k}\right) \oplus U\left(m_{k}-n_{k}\right) \rightarrow U\left(m_{k}\right),
$$

мы получаем новое асимптотическое представление $\bar{\sigma}=\left\{\bar{\sigma}_{k}\right\}$ по отношению к последовательности $\left\{m_{k}\right\}$, которое мы называем стабилизацией асимптотического представления $\sigma$.

В работе [1] было показано, что такое определение эквивалентно определению, данному в работах [2], [3]. Оно не зависит от выбора конечного подмножества $F$ при условии, что это подмножество достаточно велико, а группа $\pi$ конечно представлена.

Два асимптотических представления $\sigma_{0}$ и $\sigma_{1}$ назьваются гомотопными, если существует семейство асимптотических представлений $\sigma_{t}=\left\{\sigma_{t, k}\right\}$ таких, что функции $\sigma_{t, k}(g)$ непрерывны для всех $g \in \pi$ и $\lim _{k \rightarrow \infty} \max _{t}\left\|\sigma_{t, k}\right\|_{F}=0$, а граничные представления $\bar{\sigma}_{0}$ и $\bar{\sigma}_{1}$ являются стабилизациями представлений $\sigma_{0}$ и $\sigma_{1}$, соответственно.

Легко видеть, что каждое асимптотическое представление гомотопно некоторому асимптотическому представлению, отвечающему заданной последовательности $\left\{n_{k}\right\}$.

Асимптотические представления в конечномерные групшы $U\left(n_{k}\right)$ можно понимать также как представления в одну бесконечную группу $U(\infty)=\lim U(n)$. Группа $U(\infty)$ действует естественньм образом в сепарабельном бесконечномерном гильбертовом пространстве $H$, а норма прямого предела совпадает с нормой этого действия в $H$.

Таким образом, определения 1.1 и 1.2 можно изменить, положив $n_{k}=\infty$. Мы будем назьвать тогда последовательность $\sigma$ асимптотическим представлениe⿻⿱乛龰⿲二丨匕 в гpynny $U(\infty)$.

Ясно, что асимптотическое представление $\sigma$ в смысле определения 1.2 порождает асимптотическое представление в группу $U(\infty)$ с помощью стабилизации каждого отображения $\left\{\sigma_{k}\right\}$. Обратно, если дано асимптотическое представление $\sigma$ в группу $U(\infty)$ и возрастающая последовательность $n_{k}$, можно определить асимптотическое представление $\widetilde{\sigma}$ в смысле определения 1.2 , стабилизация которого гомотопна асимптотическому представлению $\sigma$. Более того, если два представления $\sigma_{1}$ 
и $\sigma_{2}$ в смысле определения 1.2 гомотопны как представления в группу $U(\infty)$, то они гомотопны также и как асимптотические представления в смысле определения 1.2.

Следующее обобщение понятия асимптотического представления представляется более полезным. Мы можем заменить группу $U(\infty)$ на ее замькание $\bar{U}(\infty)$ по операторной норме гильбертова пространства $H$. В этом случае остается верной стабильная гомотопическая эквивалентность между представлениями в смысле определения 1.2 и представлениями в группу $\bar{U}(\infty)$.

1.2. Асимптотическая $C^{*}$-алгебра. Хорошо известно, что асимптотические представления задают настоящие представления, однако в некоторых специальных $C^{*}$-алгебрах [3], [4]. Напомним эту конструкцию. Пусть $M_{n}=M_{n}(\mathbb{C})$ - комплексная $(n \times n)$-матричная алгебра. Зафиксируем строго возрастающую последовательность $n_{k}$. Мы будем предполагать, что матричная алгебра $M_{n_{k}}$ естественным образом вложена в алгебру $M_{n_{k+1}}$ :

$$
M_{n_{k}} \ni v \mapsto v \oplus \mathbf{0}_{n_{k+1}-n_{k}} \in M_{n_{k}} \oplus M_{n_{k+1}-n_{k}} \subset M_{n_{k+1}}
$$

Обозначим через $M_{f}$ последовательность матричных алгебр $\left\{M_{n_{k}}\right\}$. Рассмотрим унитальную $C^{*}$-алгебру

$$
B=B\left(M_{f}\right)=\prod_{k=1}^{\infty} M_{n_{k}}
$$

состоящую из ограниченных по норме последовательностей матриц. Обозначим через $B^{+} C^{*}$-алгебру $B$ с присоединенной (новой) единицей. Обе алгебры $B$ и $B^{+}$ содержат $C^{*}$-идеал

$$
I=\bigoplus_{k=1}^{\infty} M_{n_{k}}
$$

состоящий из последовательностей матрищ, нормы которых стремятся к нулю.

Пусть $Q=B / I$ и $Q^{+}=B^{+} / I-$ соответствуюшие факторалгебры. Обозначим отображения факторизации через

$$
q: B \rightarrow Q, \quad q^{+}: B^{+} \rightarrow Q^{+}
$$

Пусть

$$
\bar{\alpha}: B^{+} \rightarrow B^{+}
$$

- отображение правого сдвига,

$$
\begin{gathered}
\bar{\alpha}\left(m_{1}, m_{2}, \ldots\right)=\left(0, m_{1}, m_{2}, \ldots\right), \quad\left(m_{i}\right) \in B \subset B^{+} \\
\bar{\alpha}(\mathbf{1})=\mathbf{1}
\end{gathered}
$$

Поскольку $\bar{\alpha}(I) \subset I$, гомоморфизм $\bar{\alpha}$ индуцирует гомоморфизм факторалгебр $\alpha: Q^{+} \rightarrow Q^{+}$. Пусть

$$
Q_{\alpha}^{+}=Q_{\alpha}^{+}\left(M_{f}\right)=\left\{q \in Q^{+}: \alpha(q)=q\right\} \subset Q^{+}
$$


- $\alpha$-инвариантная $C^{*}$-подалгебра (отметим, что отображение $\alpha$ не является автоморфизмом, тем не менее его инвариантное подмножество является $C^{*}$-алгеброй). $C^{*}$-алгебра

$$
Q_{\alpha}=Q_{\alpha}\left(M_{f}\right)=\{q \in Q: \alpha(q)=q\} \subset Q
$$

определяется аналогично.

Обе алгебры $Q_{\alpha}$ и $Q_{\alpha}^{+}$являются факторалгебрами своих прообразов

$$
\begin{aligned}
B_{\alpha} & =q^{-1}\left(Q_{\alpha}\right), \\
B_{\alpha}^{+} & =\left(q^{+}\right)^{-1}\left(Q_{\alpha}^{+}\right) .
\end{aligned}
$$

Отметим, что множество $B_{\alpha}$ состоит из таких последовательностей

$$
\left(m_{1}, m_{2}, \ldots, m_{i}, \ldots\right) \in B \subset B^{+}
$$

что

$$
\lim \left\|m_{i+1}-m_{i}\right\|=0
$$

и является $C^{*}$-алгеброй.

Элементы алгебры $Q_{\alpha}^{+}$мы будем записьвать в виде $\left(\lambda,\left(m_{i}\right)\right)$, где $\lambda \in \mathbb{C}$, $\left(m_{i}\right) \in Q_{\alpha}$. Присоединенная единица дает расшепляющуюся короткую точную последовательность

$$
Q_{\alpha} \rightarrow Q_{\alpha}^{+} \stackrel{\epsilon}{\rightarrow} \mathbb{C}
$$

где $\epsilon$ - аугментация, ставящая в соответствие элементу алгебры $Q_{\alpha}^{+}$его первую компоненту.

Тогда асимптотическое представление $\sigma$ определяет гомоморфизм

$$
\bar{\sigma}: \pi \rightarrow Q_{\alpha}^{+}
$$

по формуле

$$
\bar{\sigma}(g)=q^{+}\left(1,\left\{\sigma_{k}(g)-\mathbf{E}_{n_{k}}\right\}\right) .
$$

Отметим, что из конечной представимости группы $\pi$ и из свойств (1.1) и (1.2) асимптотического представления $\sigma$ следует, что $\bar{\sigma}(g) \in Q_{\alpha}^{+}$для всех $g \in \pi$.

Ясно, что композиция

$$
\epsilon \circ \bar{\sigma}: \pi \rightarrow Q_{\alpha}^{+} \rightarrow \mathbb{C}
$$

отображает всю группу $\pi$ в единицу.

Гомоморфизм (1.4) естественным образом продолжается до унитального $*$-гомоморфизма алгебр

$$
\bar{\sigma}: C^{*}[\pi] \rightarrow Q_{\alpha}^{+},
$$

где $C^{*}[\pi]$ - групповая $C^{*}$-алгебра группы $\pi$. 
Обратно, рассмотрим унитальный *-гомоморфизм (1.5), композиция которого с аугментацией $\epsilon$ является тривиальным гомоморфизмом $C^{*}[\pi] \rightarrow \mathbb{C}$, порожденным гомоморфизмом групшы $\pi$ в тривиальную группу $е$. Тогда его ограничение на группу $\pi \subset C^{*}[\pi]$ порождается асимптотическим представлением группы $\pi$.

Аналогичньм образом, заменяя алгебры матриц на алгебру компактных операторов и рассматривая их бесконечное прямое произведение, можно определить $C^{*}$-алгебры

$$
Q_{\alpha}^{+}(\operatorname{Comp}(H))=B_{\alpha}^{+}(\operatorname{Comp}(H)) / I,
$$

где $\operatorname{Comp}(H)-C^{*}$-алгебра компактных операторов.

Таким образом, имеются следующие естественные гомоморфизмы, составляющие коммутативную диаграмму:

$$
\begin{array}{ccc}
Q_{\alpha}^{+}\left(M_{f}\right) & \rightarrow & Q_{\alpha}^{+}(\operatorname{Comp}(H)) \\
\cup & & \cup \\
Q_{\alpha}\left(M_{f}\right) & \rightarrow & Q_{\alpha}(\operatorname{Comp}(H))
\end{array}
$$

порожденную вложением алгебр

$$
M_{n_{k}} \subset \operatorname{Comp}(H)
$$

ЛЕмма 1.3. Горизонтальные стрелки диаграммы (1.6) являются гомотопическими әквивалентностями $C^{*}$-алгебр.

1.3. Вычисление $K$-групп. По лемме 1.3 при вычислении $K$-групп достаточно ограничиться лишь одной версией алгебр, которые мы будем обозначать для краткости через

$$
\begin{array}{ll}
B_{\alpha}^{+}=B_{\alpha}^{+}\left(M_{f}\right), & B_{\alpha}=B_{\alpha}\left(M_{f}\right), \\
Q_{\alpha}^{+}=Q_{\alpha}^{+}\left(M_{f}\right), & Q_{\alpha}=Q_{\alpha}\left(M_{f}\right) .
\end{array}
$$

Пусть $\mathbf{e}=\left\{\left(e_{k}\right)\right\} \in B$ - последовательность диагональных матриц, имеющих единицу на первом месте и нули на остальных, $e_{k} \in M_{n_{k}}$.

Ясно, что $\mathbf{e} \in B_{\alpha}$ и, кроме того, является проектором.

Другой проектор $\mathbf{1} \in B_{\alpha}^{+}$, являющийся единицей алгебры $B_{\alpha}^{+}$, может быть представлен как последовательность $\left(1,\left\{p_{k}=0\right\}\right)$.

ЛЕмма 1.4. Имеют место изоморфизмь

$$
\begin{aligned}
& K_{0}\left(Q_{\alpha}^{+}\right) \cong \mathbb{Z} \oplus \mathbb{Z} \text { с образующ, ими }[\mathbf{e}] u[\mathbf{1}] \\
& K_{1}\left(Q_{\alpha}^{+}\right)=0 .
\end{aligned}
$$


ДокАЗАТЕЛЬСтво. Построим гомоморфизм

$$
\operatorname{tr}=\operatorname{tr}_{1} \oplus \operatorname{tr}_{2}: K_{0}\left(Q_{\alpha}^{+}\right) \rightarrow \mathbb{Z} \oplus \mathbb{Z}
$$

Пусть $p \in M_{r}\left(Q_{\alpha}^{+}\right)$- произвольньй проектор, $p^{\prime} \in M_{r}\left(B^{+}\right)$- проектор, являющийся поднятием $p$. Такое поднятие $p^{\prime}$ существует. Действительно, пусть

$$
\begin{gathered}
p^{\prime}=\left(\lambda,\left\{p_{k}^{\prime}\right\}\right) \in M_{r}\left(B^{+}\right), \\
p^{\prime \prime}=\left\{p_{k}^{\prime}\right\} \in M_{r}(B), \\
p_{k}^{\prime} \in M_{r}\left(M_{n_{k}}\right)=M_{n_{k}}\left(M_{r}\right), \quad \lambda \in M_{r},
\end{gathered}
$$

- произвольное поднятие проектора $p \in M_{r}\left(Q_{\alpha}^{+}\right)$. Условие $p^{\prime} \in\left(q^{+}\right)^{-1}\left(Q_{\alpha}^{+}\right)=B_{\alpha}^{+}$ означает, что

$$
\left\|p_{k}^{\prime} \oplus 0 \cdot \mathbf{E}_{\left(n_{k+1}-n_{k}\right)}-p_{k+1}^{\prime}\right\| \rightarrow 0 .
$$

Поскольку $p^{2}=p$, имеем $q^{+}\left(\left(p^{\prime}\right)^{2}-p^{\prime}\right)=0$. Следовательно,

$$
\lim \left\|\left(\left(\lambda, p_{k}^{\prime}\right)\right)^{2}-\left(\lambda, p_{k}^{\prime}\right)\right\|=0
$$

Поэтому

$$
\lambda^{2}-\lambda=0
$$

и

$$
\lim \left\|\left(\left(\lambda \mathbf{E}_{n_{k}}+p_{k}^{\prime}\right)\right)^{2}-\left(\lambda \mathbf{E}_{n_{k}}+p_{k}^{\prime}\right)\right\|=0
$$

Это значит, что, начиная с номера $k$, спектр матриц $\lambda \mathbf{E}_{n_{k}}+p_{k}^{\prime}$ располагается в малой окрестности $D_{k}$ нуля и единицы, $D_{k}=D_{k}^{0} \cup D_{k}^{1}$, и

$$
\operatorname{diam}\left(D_{k}^{i}\right) \rightarrow 0
$$

Положим

$$
\tilde{p}_{k}^{\prime}=-\lambda \mathbf{E}_{n_{k}}+\int_{\gamma}\left(\lambda \mathbf{E}_{n_{k}}+p_{k}^{\prime}-\mu\right)^{-1} d \mu,
$$

где $\gamma$ - замкнутая кривая, охватывающая области $D_{k}^{1}$ и не пересекающаяся с $D_{k}^{0}$, например, $\gamma=\left\{\mu \in \mathbb{C}:|\mu-1|=\frac{1}{2}\right\}$.

Ясно, что матрица $\tilde{p}_{k}^{\prime}+\lambda \mathbf{E}_{n_{k}}$, определенная интегралом $(1.10)$, является проектором, для которого

$$
\left\|\tilde{p}_{k}^{\prime}-p_{k}^{\prime}\right\|=\left\|\left(\tilde{p}_{k}^{\prime}+\lambda \mathbf{E}_{n_{k}}\right)-\left(p_{k}^{\prime}+\lambda \mathbf{E}_{n_{k}}\right)\right\| \rightarrow 0
$$

(это следует из (1.9)). Таким образом, последовательность

$$
\tilde{p}^{\prime}=\left(\lambda,\left\{\tilde{p}_{k}^{\prime}\right\}\right)
$$

также является проектором и одновременно поднятием элемента $p$. 
Положим

$$
\begin{aligned}
& \operatorname{tr}_{1}(p)=\operatorname{tr}(\lambda) \in \mathbb{Z} \\
& \operatorname{tr}_{2}(p)=\lim _{k \rightarrow \infty} \operatorname{tr}\left(p_{k}^{\prime}\right)
\end{aligned}
$$

Предел в (1.12) существует, так как благодаря условию (1.8) имеем

$$
\lim _{k \rightarrow \infty}\left(\operatorname{tr}\left(p_{k}^{\prime}\right)-\operatorname{tr}\left(p_{k+1}^{\prime}\right)\right)=0 .
$$

С другой стороны, благодаря (1.10) имеем

$$
\left\|\tilde{p}_{k+1}^{\prime}-\tilde{p}_{k}^{\prime} \oplus 0 \cdot \mathbf{E}_{\left(n_{k+1}-n_{k}\right)}\right\| \rightarrow 0
$$

т.e.

$$
\operatorname{tr}\left(\tilde{p}_{k+1}^{\prime}\right)-\operatorname{tr}\left(\tilde{p}_{k}^{\prime}\right) \rightarrow 0 .
$$

Так как матрица $\tilde{p}_{k}^{\prime}$ является разностью двух проекторов, ее след принимает целочисленные значения. Следовательно, из (1.13) вытекает сушествование предела $\lim _{k} \operatorname{tr}\left(\tilde{p}_{k}^{\prime}\right)$ и, соответственно, существование предела в формуле (1.12).

Отметим, что определения (1.11) и (1.12) корректны, т.е. не зависят от выбора проектора $p$, представляюшего элемент $K$-группы, и от выбора проектора - поднятия $\tilde{p}^{\prime}$.

Покажем, что гомоморфизм (1.7) является изоморфизмом. Заметим, что, начиная с некоторого $k$, проекторы $\tilde{p}_{k+1}^{\prime}+\lambda \mathbf{E}_{n_{k+1}}$ и $\left(\tilde{p}_{k}^{\prime}+\lambda \mathbf{E}_{n_{k}}\right) \oplus \lambda \mathbf{E}_{\left(n_{k+1}-n_{k}\right)}$ эквивалентны, т.е.

$$
\tilde{p}_{k+1}^{\prime}+\lambda \mathbf{E}_{n_{k+1}}=U_{k+1}^{-1}\left(\left(\tilde{p}_{k}^{\prime}+\lambda \mathbf{E}_{n_{k}}\right) \oplus \lambda \mathbf{E}_{\left(n_{k+1}-n_{k}\right)}\right) U_{k+1},
$$

а унитарные матрицы $U_{k}$ могут быть выбраны сходящимися к единичному оператору

$$
\left\|U_{k}-\mathbf{E}\right\| \rightarrow 0 .
$$

Переходя к стабилизации, имеем

$$
\begin{aligned}
\left(\tilde{p}_{k+1}^{\prime}\right. & \left.+\lambda \mathbf{E}_{n_{k+1}}\right) \oplus \lambda \mathbf{E}_{\left(n_{k+s}-n_{k+1}\right)} \\
= & \left(U_{k+1} \oplus \mathbf{E}_{\left(n_{k+s}-n_{k+1}\right)}\right)^{-1} \\
& \cdot\left(\left(\tilde{p}_{k}^{\prime}+\lambda \mathbf{E}_{n_{k}}\right) \oplus \lambda \mathbf{E}_{\left(n_{k+s}-n_{k}\right)}\right)\left(U_{k+1} \oplus \mathbf{E}_{\left(n_{k+s}-n_{k+1}\right)}\right) \\
= & V_{k+1}^{-1}\left(\tilde{p}_{k}^{\prime}+\lambda \mathbf{E}_{n_{k}}\right) V_{k+1}
\end{aligned}
$$

следовательно,

$$
\begin{aligned}
\tilde{p}_{k+s}^{\prime}+ & \lambda \mathbf{E}_{n_{k+s}} \\
= & U_{k+s}^{-1}\left(U_{k+s-1} \oplus \mathbf{E}_{\left(n_{k+s}-n_{k+s-1}\right)}\right)^{-1} \cdots\left(U_{k+1} \oplus \mathbf{E}_{\left(n_{k+s}-n_{k+1}\right)}\right)^{-1} \\
& \cdot\left(\left(\tilde{p}_{k}^{\prime}+\lambda \mathbf{E}_{n_{k}}\right) \oplus \lambda \mathbf{E}_{\left(n_{k+s}-n_{k}\right)}\right) \\
& \cdot\left(U_{k+1} \oplus \mathbf{E}_{\left(n_{k+s}-n_{k+1}\right)}\right) \cdots\left(U_{k+s-1} \oplus \mathbf{E}_{\left(n_{k+s}-n_{k+s-1}\right)}\right) U_{k+s} \\
= & V_{k+s}^{-1}\left(\left(\tilde{p}_{k}^{\prime}+\lambda \mathbf{E}_{n_{k}}\right) \oplus \lambda \mathbf{E}_{\left(n_{k+2}-n_{k}\right)}\right) V_{k+s} .
\end{aligned}
$$


Последовательность $V=\left\{V_{k+s}\right\}$ принадлежит алгебре $B_{\alpha}$, поскольку

$$
V_{k+s+1}-V_{k+s}=V_{k+s}\left(U_{k+s+1}-1\right) \rightarrow 0, \quad s \rightarrow \infty .
$$

Значит,

$$
\left(1,\left\{V_{k+s}-\mathbf{E}_{n_{k+s}}\right\}\right) \in B_{\alpha}^{+} .
$$

Таким образом, проектор $\tilde{p}^{\prime}$ эквивалентен проектору $p^{\prime \prime}=\left(\lambda,\left\{p_{k+s}^{\prime \prime}\right\}\right)$, где $p_{k+s}^{\prime \prime}$ задается последовательностью с совпадающими членами:

$$
p_{k+s}^{\prime \prime}=\left(\tilde{p}_{k}^{\prime} \oplus 0 \cdot \mathbf{E}_{\left(n_{k+s}-n_{k}\right)}\right)
$$

Поэтому проектор $p^{\prime \prime}$ равен прямой сумме двух слагаемых, первое из которых, $\lambda$, эквивалентно некоторому кратному проектора $\mathbf{1}$, а второе эквивалентно некоторому кратному проектора $\mathbf{e}$.

Поскольку

$$
\begin{array}{ll}
\operatorname{tr}_{1}(\mathbf{1})=1, & \operatorname{tr}_{2}(\mathbf{1})=0 \\
\operatorname{tr}_{1}(\mathbf{e})=0, & \operatorname{tr}_{2}(\mathbf{e})=1
\end{array}
$$

имеем как сюръективность, так и инъективность гомоморфизма (1.7).

Перейдем теперь к вычислению групшы $K_{1}\left(Q_{\alpha}^{+}\right)=0$. Нам следует показать, что группа обратимых элементов матричной алгебры $M_{r}\left(Q_{\alpha}^{+}\right)$имеет только одну компоненту связности. Пусть $u=\left(\lambda,\left\{u_{k}\right\}\right) \in M_{r}\left(Q_{\alpha}^{+}\right)$- обратимый элемент. Тогда $\lambda \in M_{r}(\mathbb{C})$ - обратимая матрица. Следовательно, имеется гомотопия к случаю, когда $\lambda=\mathbf{E}_{r}$.

Действительно, пусть $\lambda_{t}$,

$$
\begin{gathered}
\operatorname{det} \lambda_{t} \neq 0, \quad 0 \leqslant t \leqslant 1, \\
\lambda_{0}=\lambda, \quad \lambda_{1}=\mathbf{E}_{r},
\end{gathered}
$$

- гомотопия матрицы $\lambda$. Тогда положим

$$
u_{t}=\left(\lambda_{t},\left\{\left(\lambda_{t} \otimes \mathbf{E}_{n_{k}}\right)\left(\lambda_{0} \otimes \mathbf{E}_{n_{k}}\right)^{-1} u_{k}\right\}\right)=\left(\lambda_{t},\left\{\left(\lambda_{t} \lambda_{0}^{-1} \otimes \mathbf{E}_{n_{k}}\right) u_{k}\right\}\right) .
$$

Легко проверить, что последовательность $\left(\lambda_{t} \lambda_{0}^{-1} \otimes \mathbf{E}_{n_{k}}\right) u_{k}$ принадлежит алгебре $M_{r}\left(B_{\alpha}\right)$ при всех $t \in[0,1]$, поэтому путь $u_{t}$ принадлежит $M_{r}\left(B_{\alpha}^{+}\right)$. Кроме того, этот путь непрерывен по $t$ и обратим.

Положим

$$
u_{1}=\left(\mathbf{E}_{r},\left\{\left(\lambda_{0}^{-1} \otimes \mathbf{E}_{n_{k}}\right) u_{k}\right\}\right)=\left(\mathbf{E}_{r},\left\{v_{k}\right\}\right),
$$

где

$$
v_{k}=\left(\lambda_{0}^{-1} \otimes \mathbf{E}_{n_{k}}\right) u_{k}
$$


Без ограничения общности можно предполагать, что элемент $u_{1} \in M_{r}\left(B_{\alpha}^{+}\right)$унитарен.

Нам нужно, далее, соединить $u_{1}$ гомотопией с единицей. Для этого рассмотрим последовательность $v_{k} \in M_{r}\left(B_{\alpha}\right)$ и выберем номер $k_{0}$ так, что $\left\|v_{k}-v_{k+1}\right\|<\frac{1}{2}$ при всех $k>k_{0}$. Будем считать, что значения $v_{k}$ являются значениями функции $v(t), 1 \leqslant t<\infty$, в целых точках $t=k$, и продолжим определение функции $v(t)$ на вешественные значения переменной $t$, выбирая выпуклую линейную комбинацию на каждом интервале $[k, k+1]$ : если $t=k+\tau, 0<\tau<1$, тогда положим

$$
v(t)=\tau v_{k+1}+(1-\tau) v_{k}
$$

Тогда легко видеть, что элемент $\mathbf{E}_{r}+v(t)$ обратим при всех $t>k_{0}$.

Требуемую гомотопию элемента $u_{1}$ можно построить по формуле

$$
u_{t}=\left(\mathbf{E}_{r},\left\{v_{k, t}\right\}\right), \quad 0 \leqslant t \leqslant 1
$$

где

$$
v_{k, t}=v\left(k_{0}+(1-t)\left(k-k_{0}\right)\right)
$$

При $t=1$ получим обратимый элемент $\left(\mathbf{E}_{r},\left\{v_{k_{0}}\right\}\right)$, задаваемый постоянной последовательностью $\left\{v_{k_{0}}\right\}$. Так же, как на первом шаге гомотопии, можно соединить элемент $\left(\mathbf{E}_{r},\left\{v_{k_{0}}\right\}\right)$ с единицей $\left(\mathbf{E}_{r},\{0\}\right)$.

1.4. Асимптотические представления как представления в алгебру Калкина. Обозначим через $\mathscr{Q}$ алгебру Калкина, т.е. факторалгебру

$$
\mathscr{Q}(H)=B(H) / \operatorname{Comp}(H),
$$

где $H$ - сепарабельное гильбертово пространство, $B(H)-C^{*}$-алгебра ограниченных операторов и $\operatorname{Comp}(H)-C^{*}$-подалгебра компактных операторов.

ТЕОРема 1.5. Имеет место вложение

$$
\psi: Q_{\alpha}^{+}(\operatorname{Comp}(H)) \otimes C\left(S^{1}\right) \rightarrow \mathscr{Q}(H)
$$

такое, что индуцированный его ограничением на подалгебру $Q_{\alpha}(\operatorname{Comp}(H)) \otimes$ $C\left(S^{1}\right)$ гомоморфизм

$$
\psi_{*}: K_{1}\left(Q_{\alpha}(\operatorname{Comp}(H)) \otimes C\left(S^{1}\right)\right) \rightarrow K_{1}(\mathscr{Q})
$$

является изоморфизмом. 
ДокАЗАТЕЛЬСтво. Начнем с определения гомоморфизма (1.14). Представим гильбертово пространство $H$ как бесконечную прямую сумму со слагаемыми, изоморфными $H$ :

$$
\widetilde{H}=\bigoplus_{k=-\infty}^{\infty} H_{k}, \quad H_{k}=H
$$

Гомоморфизм $\psi$ будет строиться как гомоморфизм в алгебру Калкина $\mathscr{Q}(\widetilde{H})$.

Рассмотрим элемент $v \in Q_{\alpha}^{+}(\operatorname{Comp}(H))$. Пусть $v^{\prime}=\left(\lambda,\left\{v_{k}^{\prime}\right\}\right) \in B_{\alpha}^{+}(\operatorname{Comp}(H))$ - поднятие элемента $v$. Определим оператор $\psi(v)$, действующий на пространстве $\widetilde{H}$ следующим образом: если $k \geqslant 1$, то оператор $\psi(v)$ действует на подпространстве $H_{k}$ как $\lambda+v_{k}^{\prime}$; если $k \leqslant 0$, то оператор $\psi(v)$ действует на подпространстве $H_{k}$ умножением на число $\lambda$.

Оператор $\psi(v)$ определен не однозначно, а с точностью до компактного слагаемого. Поэтому $\psi(v)$ корректно определяет элемент $\bar{\psi}(v) \in \mathscr{Q}(\widetilde{H})$.

Положим $\psi\left(v \otimes 1_{C\left(S^{1}\right)}\right)=\bar{\psi}(v)$. Остается определить значение $\psi$ на функции $u=e^{2 \pi i t} \in C\left(S^{1}\right)$. Рассмотрим фредгольмов оператор $F$ (индекса 0 ) в гильбертовом пространстве $\widetilde{H}$, действуюший как оператор сдвига:

$$
F: H_{k+1} \stackrel{\equiv}{\rightarrow} H_{k}
$$

Оператор $F$ коммутирует с образом $\psi\left(Q_{\alpha}^{+}(\mathrm{Comp}(H))\right)$ по модулю компактных операторов. Следовательно, мы будем иметь корректно определенный гомоморфизм $\psi$, положив

$$
\psi\left(1_{Q_{\alpha}^{+}} \otimes e^{2 \pi i t}\right)=F .
$$

Покажем теперь, что гомоморфизм (1.15) является изоморфизмом. Поскольку $K_{1}\left(Q_{\alpha}^{+}(\mathrm{Comp}(H))\right)=0$, по формуле Кюннета получаем, что

$$
K_{1}\left(Q_{\alpha}^{+}(\operatorname{Comp}(H)) \otimes C\left(S^{1}\right)\right) \cong K_{0}\left(Q_{\alpha}^{+}(\operatorname{Comp}(H))\right) \otimes K^{1}\left(S^{1}\right) .
$$

Пусть $[u] \in K^{1}\left(S^{1}\right)$ - образующая. Тогда группа $K_{1}\left(Q_{\alpha}^{+}(\operatorname{Comp}(H)) \otimes C\left(S^{1}\right)\right)$ порождается двумя элементами: $[e] \otimes[u]$ и $[1] \otimes[u]$.

Так как

$$
\psi(1 \otimes u)=F \quad \text { и } \quad \text { ind } F=0,
$$

имеем

$$
\psi_{*}([1] \otimes[u])=0 .
$$

Вычислим элемент $\psi_{*}([e] \otimes[u])$. Согласно [5] имеем

$$
[e] \otimes[u]=[(1-e) \otimes 1+e \otimes u],
$$

следовательно,

$$
\begin{aligned}
\psi_{*}([e] \otimes[u]) & =\psi_{*}([(1-e) \otimes 1+e \otimes u]) \\
& =[1-\psi(e \otimes 1)+\psi(e \otimes u)]=[1-\psi(e)+\psi(e) F] .
\end{aligned}
$$


Поскольку элемент $e \in Q_{\alpha}$ является проектором, а $\psi(e)$ коммутирует с $F$ по модулю компактных операторов, имеет место равенство

$$
\psi_{*}([e] \otimes[u])=[1-\psi(e)+\psi(e) F \psi(e)]
$$

Таким образом, оператор $G$ в правой части (1.16) является прямой суммой двух операторов: первьй из них является тождественньм на образе проектора $1-\psi(e)$, а второй - ограничение $F^{\prime}$ оператора $F$ на образ проектора $\psi(e)$. Легко проверить, что индекс оператора $F^{\prime}$ равен единице. Действительно, образ проектора $\psi(e)$ разлагается в прямую сумму одномерных подпространств, порожденных первыми базисньми векторами $e_{1, k}$ в гильбертовых пространствах $H_{k}$ при $k \geqslant 1$.

$\mathrm{C}$ другой стороны, оператор $F^{\prime}$ является оператором сдвига, переводящим вектор $e_{1, k}$ в вектор $e_{1, k-1}$. Отсюда следует, что ind $F^{\prime}=1$.

Таким образом, ind $G=1$, значит, гомоморфизм $\psi_{*}$ отображает образующую групшы $K_{1}\left(Q_{\alpha}(\operatorname{Comp}(H)) \otimes C\left(S^{1}\right)\right) \cong \mathbb{Z}$ в образующую группы $K_{1}(\mathscr{Q})$.

Обозначим через $\mathscr{R}_{a}(\pi)$ группу Гротендика асимптотических представлений группы $\pi$. Пусть $\widetilde{\mathscr{R}}_{a}(\pi)-$ ядро отображения

$$
\mathscr{R}_{a}(\pi) \rightarrow \mathscr{R}_{a}(e) \cong \mathbb{Z}
$$

определенного ограничением представления на тривиальную подгруппу $e$.

Пусть $B \pi$ - классифицируюшее пространство группы $\pi$. Напомним, что в работе [1] дана конструкция, позволяющая по асимптотическому представлению группы $\pi$ построить векторное расслоение над $B \pi$. Эта конструкция определяет гомоморфизм

$$
\varphi: \widetilde{\mathscr{R}}_{a}(\pi) \rightarrow K^{0}(B \pi)
$$

которьй можно описать следующим образом. Пусть $C^{*}[\pi]$ - групповая $C^{*}$-алгебра группы $\pi$, а $\xi \in K_{C^{*}[\pi]}^{0}(B \pi)$ - универсальное расслоение. Асимптотическое представление $\sigma$ определяет гомоморфизм

$$
\bar{\sigma}: C^{*}[\pi] \rightarrow Q_{\alpha}^{+}
$$

отображаюший универсальное расслоение $\xi$ в элемент $\bar{\sigma}_{*}(\xi) \in K_{Q_{\alpha}^{+}}^{0}(B \pi)$. Гомоморфизм (1.5) задает гомоморфизм

$$
\varphi^{\prime}: \mathscr{R}_{a}(\pi) \rightarrow K_{Q_{\alpha}^{+}}^{0}(B \pi)
$$

по формуле

$$
\varphi^{\prime}(\sigma)=\bar{\sigma}_{*}(\xi) .
$$

Рассмотрим коммутативную диаграмму

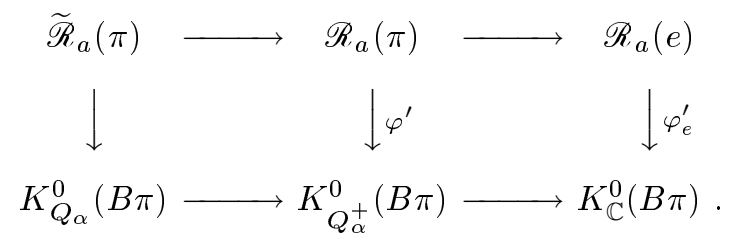


Из леммы 1.4, формулы Кюннета и из (1.3) следует, что нижняя строчка диаграммы точна, поэтому корректно определена левая вертикальная стрелка. Поскольку

$$
K_{Q_{\alpha}}^{0}(B \pi)=K^{0}(B \pi) \otimes K_{0}\left(Q_{\alpha}\right) \cong K^{0}(B \pi),
$$

мы можем определить $\varphi$ как отображение, задаваемое левой вертикальной стрелкой после отождествления $K_{Q_{\alpha}}^{0}(B \pi)$ с $K^{0}(B \pi)$. Заметим, что образ гомоморфизма $\varphi_{e}^{\prime}$ совпадает с подгруппой в $K^{0}(B \pi)$, порожденной тривиальными представлениями.

Поскольку для любой $C^{*}$-алгебры $A$ имеет место естественньй изоморфизм

$$
j: K_{A}^{0}\left(B \pi \times S^{1} \times S^{1}\right) \stackrel{\sim}{\longrightarrow} K_{A \otimes C\left(S^{1}\right)}^{0}\left(B \pi \times S^{1}\right),
$$

умножение на элемент Ботта $\beta \in K^{0}\left(S^{1} \times S^{1}\right)$ задает вложение

$$
\begin{aligned}
\bar{\beta}_{A}: K_{A}^{0}(B \pi) \stackrel{\otimes \beta}{\longrightarrow} K_{A}^{0}\left(B \pi \times S^{1} \times S^{1}\right) \stackrel{j}{\longrightarrow} K_{A \otimes C\left(S^{1}\right)}^{0}\left(B \pi \times S^{1}\right) \\
=K_{A \otimes C\left(S^{1}\right)}^{0}(B(\pi \times \mathbb{Z})) .
\end{aligned}
$$

В случае $A=Q_{\alpha}$ мы будем писать $\bar{\beta}$ вместо $\bar{\beta}_{Q_{\alpha}}$.

Обозначим через $\mathscr{R}_{\mathscr{Q}}(\pi)$ группу (виртуальных) представлений группы $\pi$ в алгебру Калкина. Легко видеть, что гомоморфизм (1.18) позволяет определить гомоморфизм

$$
\bar{\psi}: \mathscr{R}_{a}(\pi) \rightarrow \mathscr{R}_{\mathscr{Q}}(\pi \times \mathbb{Z})
$$

формулой $\bar{\psi}(\bar{\sigma})=\psi(\bar{\sigma} \otimes \mathrm{id})$ для $\bar{\sigma} \in \mathscr{R}_{a}(\pi)$ и

$$
\bar{\sigma} \otimes \mathrm{id}: C^{*}[\pi \times \mathbb{Z}] \cong C^{*}[\pi] \otimes C\left(S^{1}\right) \rightarrow Q_{\alpha}^{+} \otimes C\left(S^{1}\right) .
$$

Пусть $\eta \in K_{C^{*}[\pi \times \mathbb{Z}]}^{0}(B(\pi \times Z))$ - универсальное расслоение над $B(\pi \times \mathbb{Z})$. Тогда сушествует также гомоморфизм

$$
f: \mathscr{R}_{\mathscr{Q}}(\pi \times \mathbb{Z}) \rightarrow K_{\mathscr{Q}}^{0}(B(\pi \times \mathbb{Z})),
$$

определенньй как образ расслоения $\eta$ над $B(\pi \times \mathbb{Z})$ при представлении в алгебру Калкина.

Все описанные гомоморфизмы (1.15)-(1.22) можно представить диаграммой

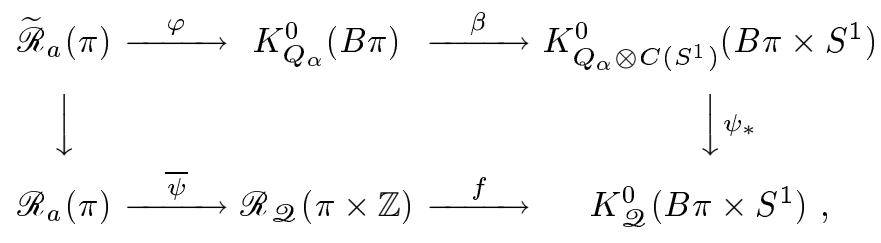

где левая вертикальная стрелка является вложением. 
Теорема 1.6. Диаграмма (1.24) коммутативна.

ДокАЗАТЕЛЬство. Непосредственное вычисление.

Диаграмма (1.24) определяет гомоморфизм

$$
\bar{\varphi}: \widetilde{\mathscr{R}}_{a}(\pi) \rightarrow K_{\mathscr{Q}}^{0}\left(B \pi \times S^{1}\right) \cong K^{1}\left(B \pi \times S^{1}\right)
$$

Теорема 1.6 также показывает, что каждый элемент группы $K^{0}(B \pi)$, который может быть получен с помощью асимптотического представления фундаментальной групшы $\pi$, может быть получен также с помощью представления группы $\pi \times \mathbb{Z}$ в алгебру Калкина, т.е. что гомоморфизм $\bar{\varphi}$ пропускается через группу $\mathscr{R} \mathscr{Q}(\pi \times \mathbb{Z})$.

\section{§ 2. Асимптотические представления и фредгольмовы представления}

Заметим, что группа $K_{\mathscr{Q}}^{0}\left(B \pi \times S^{1}\right)$ может быть разложена в прямую сумму

$$
K_{\mathscr{Q}}^{0}\left(B \pi \times S^{1}\right)=K_{\mathscr{Q}}^{0}(B \pi) \oplus K_{\mathscr{Q}}^{0}\left(B \pi \wedge S^{1}\right) \cong K^{1}(B \pi) \oplus K^{0}(B \pi)
$$

индуцированную вложением $i: s_{0} \rightarrow S^{1}$, где $s_{0} \in S^{1}$ - отмеченная точка, и образ гомоморфизма $\bar{\varphi}(1.25)$ лежит только во втором слагаемом (2.1). Действительно, рассмотрим композицию отображения $\bar{\varphi}$ с отображением $i^{*}: K_{\mathscr{Q}}^{0}\left(B \pi \times S^{1}\right)$ $\rightarrow K_{\mathscr{Q}}^{0}(B \pi)$. Поскольку в отображении $\bar{\varphi}$ участвует умножение на элемент Ботта, его композиция с $i^{*}$ есть нулевое отображение. Поэтому образ групшы $\widetilde{\mathscr{R}}_{a}(\pi)$ лежит в $K^{0}(B \pi)$ и, следовательно, определяет (виртуальное) векторное расслоение над $B \pi$.

С другой стороны, образ отображения (1.23) не обязан содержаться во втором слагаемом (2.1). Это, однако, имеет место в случае, когда представление группы $\pi \times \mathbb{Z}$ в алгебру Калкина является частью фредгольмова представления группы $\pi$ [6]. Используя понятие асимптотического представления, мы можем теперь дать обобщение фредгольмовых представлений, которое также обеспечит включение образа таких представлений во второе слагаемое (2.1).

Пусть $\rho: \pi \times \mathbb{Z} \rightarrow \mathscr{Q}$ - представление в алгебру Калкина, а $F \subset \pi$-конечное подмножество групшы $\pi$, содержащее ее образующие. Пусть $B(H)-C^{*}$-алгебра ограниченных операторов сепарабельного гильбертова пространства $H$. Обозначим через $q: B(H) \rightarrow \mathscr{Q}$ каноническую проекцию.

ОПРЕДЕЛЕНИЕ 2.1. Назовем отображение $\tau: \pi \rightarrow B(H) \varepsilon-$-тривиализацией для $\rho$, если

i) $\|\tau(g h)-\tau(g) \tau(h)\| \leqslant \varepsilon$ для всех $g, h \in F \subset \pi$;

ii) $q(\tau(g))=\rho(g ; 0)$ для всех $g \in \pi,(g ; 0) \in \pi \times \mathbb{Z}$. 
ОПРЕДЕЛЕНИЕ 2.2. Предположим, что для каждого $\varepsilon>0$ сушествует $\varepsilon$-тривиализация $\tau_{\varepsilon}$ для $\rho$. Тогда пара $\left(\tau_{\varepsilon}, \rho\right)$ называется асимптотическим фредгольмовым представлением.

Пусть $u$ - образуюшая групшы $\mathbb{Z}$. Заметим, что образ групшы $\pi$ при $\varepsilon$-тривиализациях коммутирует с некоторым фредгольмовым оператором $F \in q^{-1}(\rho(0, u)) \subset$ $B(H)$ с точностью до компактных операторов. Обозначим группу всех асимптотических фредгольмовых представлений группы $\pi$ через $\mathscr{R}_{a F}(\pi) \subset \mathscr{R}_{\mathscr{Q}}(\pi \times \mathbb{Z})$.

ПРЕДЛОЖЕНИЕ 2.3. Образ $\mathscr{R}_{a F}(\pi)$ при отображении $f$ (1.23) лежит в zpynne $K^{0}\left(B \pi \wedge S^{1}\right)$.

ДокАЗАТЕЛЬСТво. Пользуясь результатами [1], можно построить локально тривиальное расслоение над $B \pi$ (точнее, над остовом пространства $B \pi$ любой размерности) со слоями, изоморфными гильбертову пространству $H$ со структурной группой $\mathrm{GL}(H)$, исходя из почти представления $\tau_{\varepsilon}$ при достаточно малых $\varepsilon$. Для этого следует построить функции склейки, действующие в слоях $\xi_{x}$,

$$
T_{g}(x): \xi_{x} \rightarrow \xi_{g x},
$$

где $g \in \pi, x \in E \pi$. Для этого нужно выбрать по одному представителю $a_{\alpha}$ в каждой орбите нульмерных симплексов пространства $E \pi$ и определить $T_{g}\left(a_{\alpha}\right)=\tau_{\varepsilon}(g)$. Возьмем произвольный нульмерный симплекс $b \in E \pi$. Тогда существует такой элемент $h \in \pi$, что $b=h\left(a_{\alpha}\right)$, и следует положить $T_{g}(b)=\tau_{\varepsilon}(g h) \tau_{\varepsilon}^{-1}(h)$. Далее эти функции склейки продолжаются по линейности на все симплексы пространства $E \pi$. Но, очевидно,

$$
q\left(T_{g}(b)\right)=q\left(\tau_{\varepsilon}(g h) \tau_{\varepsilon}^{-1}(h)\right)=q\left(\tau_{\varepsilon}(g)\right)=\rho(g, 0),
$$

значит, после перехода к факторам функции склейки $q\left(T_{g}(x)\right)=\rho(g, 0)$ становятся постоянными, и мы получаем локально плоское расслоение над $B \pi$ с группой обратимых элементов алгебры Калкина в качестве структурной группы. Но так как любое локально тривиальное расслоение со слоем $H$ тривиально, соответствуюшее факторрасслоение со слоями, изоморфными алгебре Калкина, также тривиально, следовательно, проекция $f(\rho)$ на первое слагаемое группы $K_{\mathscr{Q}}^{0}(B \pi)$ в разложении (2.1) равна нулю.

ЗАмечАниЕ. В $\S 1$ мы видели, что асимптотическое представление $\sigma=\left(\sigma_{n}\right)$ задает гомоморфизм $\rho: \pi \times \mathbb{Z} \rightarrow \mathrm{GL}(\mathscr{Q})$ в группу обратимых элементов алгебры Калкина. Но то же самое асимптотическое представление дает также $\varepsilon$-тривиализации для $\rho$ для каждого $\varepsilon>0$. Действительно, мы можем определить их как образ в $B(H)$ последовательности почти представлений $\left(1,1, \ldots, 1, \sigma_{n}(g), \sigma_{n+1}(g), \ldots\right)$, т.е. как блочно-диагональные операторы

$$
\tau_{\varepsilon_{n}}(g)=\mathbf{E} \oplus \mathbf{E} \oplus \cdots \oplus \mathbf{E} \oplus \sigma_{n}(g) \oplus \sigma_{n+1}(g) \oplus \cdots, \quad g \in \pi .
$$

Таким образом, асимптотические представления определяют асимптотические фредгольмовы представления, и имеет место вложение $\mathscr{R}_{a}(\pi) \subset \mathscr{R}_{a F}(\pi)$. 


\section{§3. Асимптотические представления и расширения}

В работе [3] показано, что, используя квазицентральные аппроксимативные единицы, можно построить асимптотические представления, исходя из расширений $C^{*}$-алгебр. В этом параграфе мы покажем, как эта конструкция связана с асимптотическими представлениями исходной $C^{*}$-алгебры.

В теореме 1.5 было построено вложение $Q_{\alpha}^{+}(\operatorname{Comp}(H)) \subset \mathscr{Q}(H)$. Покажем, как строится аналогичное вложение $\psi: Q_{\alpha}^{+}\left(M_{f}\right) \rightarrow \mathscr{Q}$. Удобство такой конструкции заключается в том, что рассматриваемые почти представления являются конечномерньми.

Пусть $V_{k}-n_{k}$-мерное гильбертово пространство со стандартньм действием алгебры $M_{n_{k}}$ и с фиксированным базисом $\left\{e_{k}^{(i)}\right\}, i \leqslant n_{k}$. Зафиксируем вложения

$$
j_{k}: V_{k-1} \rightarrow V_{k}
$$

переводящие векторы $e_{k-1}^{(i)}$ в $e_{k}^{(i)}$. Обозначим через $W_{k-1}$ ортогональные дополнения

$$
V_{k}=j_{k}\left(V_{k-1}\right) \oplus W_{k-1}
$$

Для $k=1$ положим $W_{0}=V_{1}$. Положим, далее,

$$
H_{k}=V_{k} \oplus\left(\bigoplus_{m} W_{k, m}\right), \quad W_{k, m} \cong W_{k}, m \in \mathbb{N}, \quad H=\bigoplus_{k} H_{k}
$$

и определим гомоморфизм $\psi C^{*}$-алгебры $Q_{\alpha}^{+}\left(M_{f}\right)$ в алгебру Калкина $\mathscr{Q}$ гильбертова пространства $H$. Пусть $q \in Q_{\alpha}^{+}\left(M_{f}\right)$, а $q^{\prime}=\left(q_{k}\right) \in B^{+}\left(M_{f}\right)$ - поднятие $q$. Обозначим через $\left(q_{k}\right)_{i j}$ матричные элементы матрицы $q_{k}$. По определению последовательности $\left(q_{k}\right)_{i i}$ стабилизируются, и эти стабилизации $\lim _{i \rightarrow \infty}\left(q_{k}\right)_{i i}$ не зависят от $k$. Обозначим значение этих пределов через $\lambda(q)$. Определим $\psi(q)$ как оператор в $H$, действуюший на подпространствах $V_{k}$ умножением на матрицы $q_{k}$, а на подпространствах $W_{k, m}$ - умножением на $\lambda(q)$. Такой оператор определен с точностью до компактных операторов, поэтому он задает элемент $\psi(q) \in \mathscr{Q}$. Рассмотрим теперь $C^{*}$-алгебру $Q_{\alpha}^{+} \otimes C\left(S^{1}\right)$ непрерывных $Q_{\alpha}^{+}$-значных функций на окружности. Вышеописанная конструкция позволяет определить гомоморфизм

$$
Q_{\alpha}^{+} \otimes C\left(S^{1}\right) \rightarrow \mathscr{Q}
$$

Положим $\psi\left(q \otimes 1_{C\left(S^{1}\right)}\right)=\psi(q)$. Тогда остается определить образ функции $u=$ $e^{2 \pi i t}$ из $C\left(S^{1}\right)$. Определим фредгольмов оператор $F$ (нулевого индекса) на гильбертовом пространстве $H$ следующими условиями:

i) $F$ отображает каждое подпространство $V_{k}$ (кроме $V_{1}$ ) на подпространство $V_{k-1} \oplus W_{k-1,1}$ так, что $F\left(e_{k}^{(i)}\right)=e_{k-1}^{(i)}$ при $i<n_{k-1}$;

ii) $F$ изоморфно отображает подпространство $V_{1}$ на $W_{0,1}$;

iii) $F$ изоморфно отображает каждое подпространство $W_{k, m}$ на подпространство $W_{k, m+1}$. 
Определенный таким образом оператор $F$ коммутирует с образом $\psi\left(Q_{\alpha}^{+}\right)$по модулю компактных операторов, следовательно, мы получим требуемый гомоморфизм $\psi$, если положим $\psi\left(1_{Q_{\alpha}^{+}} \otimes e^{2 \pi i t}\right)=F$.

Обозначим базисные векторы подпространств $W_{k, m}$ через $w_{k, m}^{(j)}$, где $n_{k-1}+1 \leqslant$ $j \leqslant n_{k}$. Тогда действие оператора $F$ можно представить диаграммой

$$
\begin{aligned}
& \ldots \leftarrow w_{1,2}^{(1)} \leftarrow w_{1,1}^{(1)} \leftarrow \mid e_{1}^{(1)} \leftarrow \quad e_{2}^{(1)} \leftarrow \quad e_{3}^{(1)} \leftarrow \ldots \\
& \begin{array}{lllllllllllll}
\ldots & \ldots & \ldots & \ldots & \ldots & \ldots & \ldots & \ldots & \ldots & \ldots & \ldots & \ldots & \ldots
\end{array} \\
& \ldots \leftarrow w_{1,2}^{\left(n_{1}\right)} \leftarrow w_{1,1}^{\left(n_{1}\right)} \leftarrow \mid e_{1}^{\left(n_{1}\right)} \leftarrow e_{2}^{\left(n_{1}\right)} \leftarrow e_{3}^{\left(n_{1}\right)} \leftarrow \ldots \\
& \ldots \leftarrow w_{2,3}^{\left(n_{1}+1\right)} \leftarrow w_{2,2}^{\left(n_{1}+1\right)} \leftarrow w_{2,1}^{\left(n_{1}+1\right)} \leftarrow \mid e_{2}^{\left(n_{1}+1\right)} \leftarrow e_{3}^{\left(n_{1}+1\right)} \leftarrow \ldots \\
& \begin{array}{lllllllllllll}
\ldots & \ldots & \ldots & \ldots & \ldots & \ldots & \ldots & \ldots & \ldots & \ldots & \ldots & \ldots & \ldots
\end{array} \\
& \ldots \leftarrow w_{2,3}^{\left(n_{2}\right)} \leftarrow w_{2,2}^{\left(n_{2}\right)} \leftarrow w_{2,1}^{\left(n_{2}\right)} \leftarrow \mid e_{2}^{\left(n_{2}\right)} \leftarrow e_{3}^{\left(n_{2}\right)} \leftarrow \ldots \\
& \ldots \leftarrow w_{3,4}^{\left(n_{2}+1\right)} \leftarrow w_{3,3}^{\left(n_{2}+1\right)} \leftarrow w_{3,2}^{\left(n_{2}+1\right)} \leftarrow w_{3,1}^{\left(n_{2}+1\right)} \leftarrow \mid e_{3}^{\left(n_{2}+1\right)} \leftarrow \ldots \\
& \begin{array}{lllllllllllll}
\ldots & \ldots & \ldots & \ldots & \ldots & \ldots & \ldots & \ldots & \ldots & \ldots & \ldots & \ldots & \ldots
\end{array} \\
& \ldots \leftarrow w_{3,4}^{\left(n_{3}\right)} \leftarrow w_{3,3}^{\left(n_{3}\right)} \leftarrow w_{3,2}^{\left(n_{3}\right)} \leftarrow \quad w_{3,1}^{\left(n_{3}\right)} \leftarrow \mid e_{3}^{\left(n_{3}\right)} \leftarrow \ldots \\
& \begin{array}{llllllllllll}
\ldots & \ldots & \ldots & \ldots & \ldots & \ldots & 3,2 & & 3,1 & & & \\
& \ldots & \ldots & \ldots & \ldots & \ldots & \ldots & \ldots & \ldots
\end{array}
\end{aligned}
$$

Пусть $A$ - такая $C^{*}$-алгебра, что существует гомоморфизм аугментации

$$
\epsilon_{A}: A \rightarrow \mathbb{C}
$$

алгебры $A$ в поле комплексных чисел, а $\mathscr{F} A \subset A$ - конечный набор образующих алгебры $A$. Мы можем повторить наши определения асимптотических представлений из $\S 1$ для случая $C^{*}$-алгебр вместо дискретных групп.

Для удобства обозначений далее мы будем считать, что размерность почти представления $\widetilde{\sigma}_{k}$ равна $k$.

Рассмотрим последовательность отображений $\widetilde{\sigma}_{k}: A \rightarrow M_{k}, \quad \widetilde{\sigma}_{k}(1)=1$, $\left\|\widetilde{\sigma}_{k}(a b)-\widetilde{\sigma}_{k}(a) \widetilde{\sigma}_{k}(b)\right\| \rightarrow 0$ для $a, b \in \mathscr{F}_{A}$, где $M_{k}$ стандартно действует в конечномерном гильбертовом пространстве $V_{k}$. Возьмем бесконечномерное гильбертово пространство $H_{k} \supset V_{k}$ и определим отображение $\sigma_{k}: A \rightarrow B\left(H_{k}\right)$ равенством

$$
\sigma_{k}(a)=\widetilde{\sigma}_{k}(a) \oplus \epsilon_{A}(a)
$$

где $a \in A$, а $\epsilon_{A}(a)$ - скалярный оператор в $V_{k}^{\perp}$. Последовательность отображений $\left\{\sigma_{k}\right\}$ задает асимптотическое представление $C^{*}$-алгебры $A$, если (после отождествления всех $\left.H_{k}\right)$ нормы $\left\|\sigma_{k+1}(a)-\sigma_{k}(a)\right\|$ стремятся к нулю для всех $a \in \mathscr{F}_{A}$.

Положим $\sigma=\bigoplus_{k} \sigma_{k}: \pi \rightarrow B(H)$, где $H=\bigoplus_{k} H_{k}$. Рассмотрим в гильбертовом пространстве $H C^{*}$-алгебру $\mathscr{E} 0$, порожденную всеми компактньми операторами, а также всеми операторами вида $\sigma(a), a \in A$, и $C^{*}$-алгебру $\mathscr{E}$, порожденную алгеброй $\mathscr{E}_{0}$ и оператором сдвига $F$, определенным выше. Эти $C^{*}$-алгебры содержат идеал $\operatorname{Comp}(H)$. Рассмотрим факторалгебру $\mathscr{E}_{0} / \operatorname{Comp}(H)$. Она изоморфна факторалгебре $C^{*}$-алгебры $A$ по некоторому идеалу $J \subset A$. Тогда, поскольку оператор $F$ коммутирует с элементами алгебры $\mathscr{E}_{0}$ по модулю компактных операторов 
и спектр $F$ совпадает с единичной окружностью, имеет место короткая точная последовательность

$$
\operatorname{Comp}(H) \rightarrow \mathscr{E} \rightarrow(A / J) \otimes C\left(S^{1}\right)
$$

Мы воспользуемся дискретной версией конструкции из работы [3]. Напомним, что аппроксимативная единица $\left\{e_{n}\right\} \in \operatorname{Comp}(H), 0 \leqslant e_{n} \leqslant 1$, называется квазицентральной (см. [7]) для короткой точной последовательности (3.1), если для любого элемента $b \in \mathscr{E}$ имеет место равенство $\lim _{n}\left\|e_{n} b-b e_{n}\right\|=0$. Точная последовательность (3.1) определяет асимптотическое представление

$$
\bar{\rho}_{n}: A \otimes C\left(S^{1}\right) \otimes C_{0}\left(S^{1}\right) \stackrel{a s}{\rightarrow} \operatorname{Comp}(H)
$$

по формуле

$$
\bar{\rho}_{n}(a \otimes g \otimes f)=(a \otimes g)^{\prime} \cdot f\left(e_{n}\right)
$$

где $(a \otimes g)^{\prime} \in \mathscr{E}$ - поднятие элемента $(a J) \otimes g \in(A / J) \otimes C\left(S^{1}\right)$, a $f \in C_{0}\left(S^{1}\right)$. Обозначим через $K_{n} \subset H$ подпространство, на котором $e_{n} \neq 0, e_{n} \neq 1$. Без ограничения общности (заменив элементы $e_{n}$ на близкие к ним элементы конечного ранга) можно считать, что подпространства $K_{n}$ конечномерны, $r(n)=\operatorname{dim} K_{n}$. Тогда мы можем определить последовательность конечномерных отображений

$$
\rho_{n}: A \otimes C\left(S^{1}\right) \otimes C_{0}\left(S^{1}\right) \stackrel{a s}{\rightarrow} M_{r(n)}
$$

по формуле

$$
\rho_{n}(a \otimes g \otimes f)=P_{n}(a \otimes g)^{\prime} P_{n} \cdot f\left(e_{n}\right)
$$

где $P_{n}$ - проекция на $K_{n}, g \in C\left(S^{1}\right), f \in C_{0}\left(S^{1}\right), f(0)=0$. Из квазицентральности $\left\{e_{n}\right\}$ следует, что для любых $a \otimes g \in A \otimes C\left(S^{1}\right), f \in C_{0}\left(S^{1}\right)$ имеем

$$
\lim _{n \rightarrow \infty}\|\bar{\rho}(a \otimes g \otimes f)-\rho(a \otimes g \otimes f)\|=0
$$

поэтому последовательность отображений (3.2) является асимптотическим представлением.

Пусть $\mathscr{F}^{\prime}=\left\{\mathscr{F}_{A} \otimes 1,1 \otimes e^{2 \pi i x}\right\}-$ конечный набор образуюших $C^{*}$-алгебры $A \otimes C\left(S^{1}\right)$. Для дальнейшего нам понадобится квазицентральная аппроксимативная единица специального вида. Определим последовательность натуральных чисел $m(n)$ так, чтобы были выполнены следующие условия:

i) $m(n)$ стремится к бесконечности;

ii) $\max _{1 \leqslant i \leqslant m(n)}\left\|\sigma_{n+i}(a)-\sigma_{n}(a)\right\|$ стремится к нулю для любого $a \in \mathscr{F}_{A} \subset A$;

iii) $\frac{m(n+1)-m(n)}{m(n)} \rightarrow 0$ при $n \rightarrow \infty$. 
Мы предположили для удобства обозначений, что $\operatorname{dim} V_{k}=k$, значит, $\operatorname{dim} W_{k, l}=\operatorname{dim} V_{k}-\operatorname{dim} V_{k-1}=1$. Переобозначим базисные векторы подпространств $V_{k}$ через $v_{k}^{(j)}, j=1, \ldots, k$, а базисные векторы подпространств $W_{k, l}$ через $v_{k-l}^{(k)}$. В этих обозначениях оператор сдвига $F$, изображенный диаграммой на стр. 69 , действует по формуле

$$
F v_{k}^{(j)}=v_{k-1}^{(j)}
$$

и является прямой суммой операторов сдвига в подпространствах $H^{(j)}$, порожденных векторами $v_{k}^{(j)}$.

Для $k \geqslant 0$ положим

$$
a_{n, k}^{(j)}= \begin{cases}1 & \text { при } k \leqslant n \text { и } j \leqslant n+m(n), \\ \frac{m(n)-k+n}{m(n)} & \text { при } n<k \leqslant n+m(n) \text { и } j \leqslant n+m(n), \\ 0 & \text { в остальных случаях, } \\ & a_{n,-k}^{(j)}=a_{n, k}^{(j)}\end{cases}
$$

и определим последовательность элементов $\left\{e_{n}\right\}$ как последовательность диагональных операторов в $H$, действующих умножением по формуле

$$
e_{n} v_{k}^{(j)}=a_{n, k}^{(j)} v_{k}^{(j)}
$$

ЛЕмма 3.1. Последовательность $\left\{e_{n}\right\}$ является квазицентральной аппроксимативной единичей для точной последовательности (3.1).

ДокАЗАТЕЛЬСтво. Легко видеть, что последовательность $\left\{e_{n}\right\}$ является аппроксимативной единицей, поскольку для всех $k, j$ имеем $e_{n} v_{k}^{(j)} \rightarrow v_{k}^{(j)}$. Покажем, что $\left\{e_{n}\right\}$ квазицентральна. Для этого достаточно оценить нормы коммутаторов

$$
\left[e_{n}, \sigma(a)\right] \text { для } a \in \mathscr{F}_{A} \text { и }\left[e_{n}, F\right] .
$$

Из определения $e_{n}$ видно, что $\left[e_{n}, \sigma(a)\right] v_{k}^{(j)}=0$ для всех $k, j$ кроме $k=j=$ $n+m(n)+1, \mathrm{a}$

$$
\left[e_{n}, \sigma(a)\right] v_{n+m(n)+1}^{(n+m(n)+1)}=e_{n}\left(\epsilon_{A}(a) v_{n+m(n)+1}^{(n+m(n)+1)}+v^{\prime}\right)-\sigma(a) e_{n} v_{n+m(n)+1}^{(n+m(n)+1)}
$$

где $v^{\prime} \in \operatorname{Span}\left\{v_{n+m(n)+1}^{(j)}\right\}, 1 \leqslant j \leqslant n+m(n)+1$, и $\left\|v^{\prime}\right\| \rightarrow 0$ при $n \rightarrow \infty$. Но так как $e_{n} v_{n+m(n)+1}^{(n+m(n)+1)}=0$, то

$$
\lim _{n \rightarrow \infty}\left\|\left[e_{n}, \sigma(a)\right] v_{n+m(n)+1}^{(n+m(n)+1)}\right\|=\lim _{n \rightarrow \infty}\left\|e_{n} v^{\prime}\right\|=0 .
$$


Для второго коммутатора имеем

$$
\lim _{n \rightarrow \infty}\left\|\left[e_{n}, F\right]\right\|=\lim _{n \rightarrow \infty}\left\|\bigoplus_{k}\left[\left.e_{n}\right|_{H_{k}},\left.F\right|_{H_{k}}\right]\right\|=\lim _{n \rightarrow \infty} \frac{1}{m(n)}=0
$$

следовательно, $\left\{e_{n}\right\}$ квазицентральна.

Отметим также, что, поскольку

$$
\left\|e_{n+1}-e_{n}\right\| \leqslant \max \left\{\frac{1}{m(n+1)} ; \frac{m(n+1)-m(n)}{m(n)}\right\} \rightarrow 0
$$

получаем $\left\|\rho_{n+1}-\rho_{n}\right\| \rightarrow 0$ на образующих алгебры $A \otimes C\left(S^{1}\right) \otimes C_{0}\left(S^{1}\right)$.

Напомним, что через $P_{n}$ мы обозначили проектор на подпространство в $H$, на котором $e_{n} \neq 0, e_{n} \neq 1$, т.е. на подпространство, порожденное векторами $v_{k}^{(j)}$ при $n<|k| \leqslant n+m(n), j \leqslant n+m(n)$. Разобьем проектор $P_{n}$ на две части: $P_{n}=P_{n}^{+} \oplus P_{n}^{-}$, где $P_{n}^{+}-$подпроектор $P_{n}$ на линейную оболочку векторов $v_{k}^{(j)}$ при $k>0$. Тогда формулы $\rho_{n}^{ \pm}=P_{n}^{ \pm} \rho P_{n}^{ \pm}$задают отображения $\rho^{ \pm}=\left\{\rho_{n}^{ \pm}\right\}$:

$$
\rho_{n}^{ \pm}: A \otimes C\left(S^{1}\right) \otimes C_{0}\left(S^{1}\right) \rightarrow M_{(n+m(n)) \times m(n)} .
$$

Ясно, что $\rho=\rho^{+} \oplus \rho^{-}$.

Известно еще одно асимптотическое представление $\beta=\left(\beta_{m}\right)$ коммутативной $C^{*}$-алгебры $C\left(S^{1} \times S^{1}\right)$ в матричные алгебры $M_{m}$, определенное формулой

$$
\beta_{m}\left(e^{2 \pi i k x} e^{2 \pi i l y}\right)=T_{m}^{k} U_{m}^{l}, \quad \beta_{m}: C\left(S^{1}\right) \otimes C\left(S^{1}\right) \stackrel{a s}{\rightarrow} M_{m},
$$

где $T_{m}$ и $U_{m}-m$-мерные матрицы Войкулеску: $T_{m}$ - оператор сдвига, а $U_{m}-$ диагональная матрица [8]. Это асимптотическое представление реализует изоморфизм Ботта.

Обратный элемент для образуюшей Ботта задается асимптотическим представлением $-\beta=(-\beta)_{m}$, где

$$
(-\beta)_{m}\left(e^{2 \pi i k x} e^{2 \pi i l y}\right)=T_{m}^{k} U_{m}^{-l} .
$$

Пусть $m=m(n)$. Тензорное произведение $\widetilde{\sigma}$ и $\beta$ дает асимптотическое представление $\widetilde{\sigma} \otimes \beta=(\widetilde{\sigma} \otimes \beta)_{n}$, где

$$
(\widetilde{\sigma} \otimes \beta)_{n}=\widetilde{\sigma}_{n} \otimes \beta_{m(n)}: A \otimes C\left(S^{1}\right) \otimes C_{0}\left(S^{1}\right) \stackrel{a s}{\rightarrow} M_{n \times m(n)} .
$$

Напомним, что через $\epsilon_{A}: A \rightarrow \mathbb{C}$ мы обозначаем гомоморфизм аугментации, и обозначим через $\epsilon_{n}$ прямую сумму $n$ экземпляров гомоморфизма $\epsilon_{A}$. Тензорное произведение $\epsilon_{A} \otimes(-\beta)=\left(\epsilon_{n} \otimes(-\beta)_{m(n)}\right)$ также определяет асимптотическое представление, тривиальное на $A$. 
ПРЕДЛОЖЕНИЕ 3.2. (i) Отображения $\rho^{+} u \rho^{-}$являются асимптотическими представлениями алгебры $A \otimes C\left(S^{1}\right) \otimes C_{0}\left(S^{1}\right)$.

(ii) Асимптотические представления $\rho^{+}$и $\widetilde{\sigma} \otimes \beta$ в матричные алгебры $M_{(n+m(n)) \times m(n)}$ являются әквивалентными; более того, для $а \in \mathscr{F}_{A} \subset A$, $e^{2 \pi i x} \in C\left(S^{1}\right)$ u $f \in C_{0}\left(S^{1}\right)$ нормиц

$$
\left\|\rho_{n}^{+}\left(a \otimes e^{2 \pi i x} \otimes f\right)-\widetilde{\sigma}_{n}(a) \otimes \beta_{m(n)}\left(e^{2 \pi i x} \otimes f\right)\right\|
$$

стремятся к нулю.

(iii) Асимптотические представления $\rho^{-} u \epsilon_{A} \otimes(-\beta)$ являются эквивалентныци; более того, для $а \in \mathscr{F}_{A} \subset A, e^{2 \pi i x} \in C\left(S^{1}\right)$ u $f \in C_{0}\left(S^{1}\right)$ норми

$$
\left\|\rho_{n}^{-}\left(a \otimes e^{2 \pi i x} \otimes f\right)-\epsilon_{n}(a) \otimes(-\beta)_{m(n)}\left(e^{2 \pi i x} \otimes f\right)\right\|
$$

стремятся к нулю.

(iv) B группе (виртуальных) асимптотических представлений имеет место равенство

$$
[\bar{\rho}]=[\rho]=[\widetilde{\sigma} \otimes \beta]-\left[\epsilon_{A} \otimes \beta\right] .
$$

ДокАЗАТЕльство. Первое утверждение предложения следует из того, что

$$
\left\|\left[f\left(e_{n}\right), P_{n}^{ \pm} \sigma(a) P_{n}^{ \pm}\right]\right\| \rightarrow 0 \text { при } a \in \mathscr{F}_{A}, \quad\left\|\left[f\left(e_{n}\right), P_{n}^{ \pm} F P_{n}^{ \pm}\right]\right\| \rightarrow 0 .
$$

Прямые вычисления показывают, что

$$
\rho_{n}\left(a \otimes e^{2 \pi i x} \otimes f\right)=P_{n}^{+}\left(\bigoplus_{i=n+1}^{m(n)} \sigma_{i}(a)\right) P_{n}^{+} F P_{n}^{+} f\left(e_{n}\right)=\bigoplus_{i=n+1}^{m(n)} \widetilde{\sigma}_{i}(a) F P_{n}^{+} f\left(e_{n}\right),
$$

где $a^{\prime}=\bigoplus_{i=1}^{\infty} \sigma_{i}(a) \in \mathscr{E}$ является поднятием для $a, F$ является поднятием для $e^{2 \pi i x}$ и $f\left(e_{n}\right)$ - диагональная матрица с элементами $f\left(\frac{m(n)-i+n}{m(n)}\right)$ на диагонали.

Обозначим (так же, как матрицу сдвига из пары матриц Войкулеску) через $T_{m(n)}$ оператор в $H$, действуюший по формуле

$$
T_{m(n)} v_{k}^{(j)}=v_{k-1}^{(j)} \text { при } n+2 \leqslant k \leqslant n+m(n), \quad T_{m(n)} v_{n+1}^{(j)}=v_{n+m(n)}^{(j)} .
$$

Тогда легко видеть, что нормы

$$
\left\|P_{n} F P_{n} f\left(e_{n}\right)-T_{m(n)} f\left(e_{n}\right)\right\|
$$

стремятся к нулю и что

$$
T_{m(n)} f\left(e_{n}\right)=\beta_{m(n)}\left(e^{2 \pi i x} \otimes f\right) .
$$

Остается заметить, что благодаря нашему выбору последовательности $m(n)$ нормы

$$
\left\|\left(\bigoplus_{i=n+1}^{m(n)} \widetilde{\sigma}_{i}\right)(a)-\left(\bigoplus_{i=n+1}^{m(n)} \widetilde{\sigma}_{n}\right)(a)\right\|
$$

также стремятся к нулю, что доказьвает утверждение (ii).

Аналогично разбирается случай асимптотического представления $\rho^{-}$. Последнее утверждение предложения очевидно следует из предыдущих. 


\section{Список литературы}

1. Mishchenko A.S., Noor Mohammad. Asymptotical representations of discrete groups // Lie Groups and Lie Algebras Their Representations, Generalizations and Applications. Mathematics and Its Applications. V.433. Dordrecht: Kluwer Academic Press, 1998. P. 299-312.

2. Connes A., Gromov M., Moscovici H. Conjecture de Novikov et fibrés presque plats // C. R. Acad. Sci. Paris. Sér. I. Math. 1990. V. 310. P. 273-277.

3. Connes A., Higson $N$. Deformations, morphismes asymptotiques et $K$-theorie bivariante // C. R. Acad. Sci. Paris. Sér. I. Math. 1990. V. 311. P. 101-106.

4. Loring T.A., Pedersen G.K. Corona extendibility and asymptotic multiplicativity // $K$-Theory. 1997. V. 11. P. 83-102.

5. Connes $A$. An analogue of the Thom isomorphism for crossed products of a $C^{*}$-algebra by an action of $\mathbb{R} / /$ Adv. Math. 1981. V. 39. P. 31-55.

6. Мищенко A.C. О фредгольмовых представлениях дискретных групп // Функц. анализ и его прилож. 1975. Т. 9. № 2. С. 36-41.

7. Arveson $W$. Notes on extensions of $C^{*}$-algebras // Duke Math. J. 1977. V. 44. P. 329-355.

8. Voiculescu D. Asymptotically commuting finite rank unitary operators without commuting approximants // Acta Sci. Math. (Szeged). 1983. V. 45. P. 429-431.

Московский государственнњй

Поступила в редакцию

университет им. М.В. Ломоносова

06.03 .1998 